This is a self-archived version of an original article. This version may differ from the original in pagination and typographic details.

Author(s): Pareja-Blanco, Fernando; Walker, Simon; Häkkinen, Keijo

Title: Validity of Using Velocity to Estimate Intensity in Resistance Exercises in Men and Women

Year: 2020

Version: Accepted version (Final draft)

Copyright: @ 2020 Georg Thieme Verlag KG

Rights: In Copyright

Rights url: http://rightsstatements.org/page//nC/1.0/?language=en

Please cite the original version:

Pareja-Blanco, F., Walker, S., \& Häkkinen, K. (2020). Validity of Using Velocity to Estimate Intensity in Resistance Exercises in Men and Women. International Journal of Sports Medicine, 41(14), 1047-1055. https://doi.org/10.1055/a-1171-2287 


\title{
VALIDITY OF USING VELOCITY TO ESTIMATE INTENSITY IN RESISTANCE EXERCISES IN MEN AND WOMEN
}

\begin{abstract}
This study aimed to examine the validity of using bar velocity to estimate relative load in squat and bench-press exercises for both young men and women. Twenty-five men and 25 women performed a progressive loading test up to 1-RM in the squat and bench-press exercises, which were repeated after 2 weeks. Relationships between mean propulsive velocity and \%1-RM were analysed. A second-order polynomial equation for predicting the corresponding MPV of each percentage of 1-RM was developed for men (validation). This equation was then applied in women (cross-validation). Moreover, a specific equation for women was developed (validation) and was also applied in a sub-sample of women (cross-validation). Close relationships $\left(\mathrm{R}^{2}: 0.91-0.95\right)$ between bar velocity and relative load were observed in both sexes for squat and bench press. Men's equation applied to women showed a high level of agreement, although lower bias and higher level of agreement was observed when a sex-specific equation was applied in women, both validation and cross-validation samples.. In conclusion, lifting velocity can be used to accurately prescribe the relative load regardless of sex in both upper-body and lowerbody exercises, although when estimating load from velocity measures it will be necessary to use the sex-specific equation for each exercise.
\end{abstract}

Key Words: loading intensity, velocity-based training, maximal strength, one-repetition maximum, athletic performance, gender 


\section{INTRODUCTION}

Resistance training has gained immense popularity over the last decades in non-athletic populations, such as children and healthy and frail elderly in both sexes. Among the different training variables that should be considered when designing resistance training programs $[1,2]$, exercise intensity seems to be a paramount parameter influencing neuromuscular adaptations [3, 4]. Therefore, a common concern of strength and conditioning professionals is how to objectively quantify and monitor the actual exercise intensity undertaken by trainees. The most common reference to prescribe exercise intensity and evaluate the individual's maximal strength level is the one-repetition maximum (1-RM, i.e. the maximum load that can be lifted successfully once) $[2,5]$. Typically, the 1-RM is used to precisely prescribe submaximal training intensities in the form of percentages of 1-RM (\%1-RM) [6]. Despite its extensive utilization, the implementation of 1-RM assessment presents important drawbacks [7, 8]. This approach is frequently described as a very time consuming and critical procedure [8,9], and it has been suggested that the test may increase injury risk when performed incorrectly [10]. Additionally, 1-RM testing can induce muscle damage and potentially deteriorate performance on subsequent days [11]. Furthermore, the 1-RM value can fluctuate on a day-to-day basis, or change throughout the training program. Consequently, there is a need to find faster and safer methods to accurately estimate 1-RM while minimizing the accumulation of fatigue. These 1-RM fluctuations could be identified using velocitybased training.

Velocity-based training (VBT) proposes that lifting velocity is a valid alternative to quantify and adjust training intensity with high precision on a daily basis. This approach is based on the extremely close relationship, originally observed in the bench press (BP) exercise, between \%1-RM and barbell velocity $\left(\mathrm{R}^{2}=0.98\right)$ [8]. Indeed, this close relationship enables coaches to accurately estimate the individual's current 1-RM performance, and, therefore, to prescribe, in real-time, the training loads during resistance training programs $[7,8]$. This issue has been studied in men using exercises such as the prone bench pull [12], pull-up [13, 14], leg-press [15], hip-thrust [16] and a number of variations of the squat (SQ) exercise $[9,17,18]$. Therefore, in these exercises, intensity can be prescribed on a daily basis by adjusting the absolute load (kg) to match the repetition velocity associated with the \%1-RM that is intended for the training session. 
Notwithstanding, as a drawback of this methodology, the load-velocity relationship in resistance exercises has been analysed almost exclusively in men. Only three previous studies have reported the load-velocity relationship in women [19-21]. These studies were conducted on upper-body exercises (BP, inclined BP, and military press) and observed that the load-velocity relationship was strong and linear (individual $\mathrm{R}^{2}$ range: $0.987-$ 0.993 ) in both sexes but the velocity associated with each \%1-RM was higher in men compared to women [19-21]. Therefore, the general equations previously published in the main exercises, such as BP and SQ $[8,9,17,18]$ may not be suitable for women. As a result, if the training load is prescribed with the same velocity value for both men and women, the training stimulus for men and women would be different. Hence, the expected outcomes may not be the same, and ultimately, it would not be possible to extrapolate findings from training studies conducted in men to women.

Importantly, if the load-velocity relationship is sensitive enough to predict the \%1-RM being employed, the stability of its predictions must first be established. To our knowledge, although the between-session reliability load-velocity relationship in men has been already explored [22, 23], the reliability of the load-velocity relationship in women has not been examined yet. Thus, in order to successfully implement the VBT approach e.g. in scientific studies and/or athletic training, it is imperative to have sufficiently accurate and reliable models for monitoring relative load. Therefore, the sex-specific equations may provide more accurate load prescription during VBT programs carried out in women. However, whether the findings from BP studies [19-21] can be extrapolated to one of the most commonly prescribed lower-body exercises in resistance training settings such as SQ, has never been tested in the literature.

As another drawback to understanding this topic, previous literature attempting to validate the use of movement velocity to estimate relative load [7-9, 12-21] has used methods based on correlation, and these methods cannot, on their own, assess systematic bias [26]. In order to assess the level of agreement between the observed and the predicted data a comprehensive set of statistics should be used [26, 27]. Despite the increasing interest in VBT, there is a lack of studies examining the validity and reliability of using lifting velocity to estimate relative load during resistance exercises for both men and women. Due to these drawbacks, current evidence about the validity of using lifting velocity to estimate relative load can be questioned. This information is a research gap that requires to be addressed to examine the validity of VBT approach to guarantee its 
suitability for monitoring actual training loads being used during resistance training interventions. Therefore, the aim of the present study was to determine the validity of using the bar velocity to estimate the relative load (\%1-RM) in upper- and lower-body exercises (BP and SQ) for both men and women.

\section{MATERIAL AND METHODS}

\section{Participants}

Twenty-five young men (age $=25.8 \pm 3.3$ years, body mass $=82.8 \pm 11.2 \mathrm{~kg}$, height $=$ $179.8 \pm 6.1 \mathrm{~cm})$ and 25 young women $($ age $=26.1 \pm 4.0$ years, body mass $=60.7 \pm 6.1$ $\mathrm{kg}$, height $=166.6 \pm 7.2 \mathrm{~cm}$ ) volunteered to take part in this study. Inclusion criteria were: 1) being physically active capable of performing a technically correct BP and SQ exercises; and 2) having at least 1 year of resistance training background. After being informed of the purpose and testing procedures, participants signed a written informed consent form prior to participation. The University Ethics Committee approved the experimental procedures of the study, which was conducted according to the Declaration of Helsinki. The study meets the ethical standards of the journal [28].

\section{Study Design}

Participants underwent a preliminary session during which they were familiarized with the testing equipment and exercise protocol. This session was used for body composition assessment, medical examination and personal data and health history questionnaire administration. During the familiarization, participants performed an incremental loading test until they could no longer produce a mean propulsive velocity (MPV) above 1.00 and $0.80 \mathrm{~m} \cdot \mathrm{s}^{-1}$ in SQ and BP respectively (approx. 50-60\%1-RM), while researchers emphasized proper technique and maximum concentric velocity. All velocity values presented in this study were derived from the MPV. The propulsive phase was defined as that portion of the concentric phase during which barbell acceleration is greater than the acceleration due to gravity $[29,30]$. One week later, all 50 participants performed progressive loading tests up to 1-RM for individual determination of the full load-velocity relationship (T1) in both SQ and BP exercises. Finally, after 2 weeks, all participants reperformed the same incremental loading tests (T2). During this 2-week control period, participants were advised to continue with their own habitual training. This third session was performed with the aim of analysing the repeatability of the load-velocity 
relationship. Testing sessions were performed at the same place and time of day for each participant, under the same environmental conditions. Participants abstained from any strenuous physical activity for at least 2 days before each testing session.

\section{Testing procedures}

A progressive loading test was performed to obtain each individual's load-velocity relationship and 1-RM strength determination using a Smith Machine in the SQ (Kraftwerk, Tuusula, Finland) and BP exercises (Marbo Sport, Starachowice, Poland), in that order. The SQ was performed starting from the upright position with the knees and hips fully extended. Each participant descended in a controlled manner until the top of the thighs were below the horizontal plane, then immediately ascended back to the upright position as fast as possible. The participants were instructed that the feet must remain in contact with the floor (i.e. no jumping) but the heels were allowed to rise up slightly in the air. A self-selected stance width (approximately 100\% of the biacromial distance) was used throughout the first testing session and the foot position was recorded in order to be repeated in the subsequent testing sessions. Warm-up consisted of 5 min of stationary cycling at a self-selected easy pace, 5 min of lower-body joint mobilisation exercises, 10 squat repetitions without external load, followed by 6 repetitions with fixed loads of 30 and $20 \mathrm{~kg}$ for men and women, respectively. The exact same warm-up and progression of submaximal loads up to the 1-RM was repeated in the post-test for each participant. After the warm-up, the initial load was set at $40 \mathrm{~kg}$ for men and $30 \mathrm{~kg}$ for women and progressively increased, by $10 \mathrm{~kg}$, until the attained MPV was $<0.5 \mathrm{~m} \cdot \mathrm{s}^{-1}$. Thereafter, each load was individually adjusted with smaller increments $(1-5 \mathrm{~kg})$ so that $1-\mathrm{RM}$ could be precisely determined. The average number of attempts below a MPV of $0.5 \mathrm{~m} \cdot \mathrm{s}^{-1}$ was $3.0 \pm 1.3$, complying with NSCA guidelines [31]

Similarly for BP, a self-selected width with pronated grip (approximately $150 \%$ of the biacromial distance) was used throughout the first testing session and the hand position was recorded in order to be repeated in the subsequent testing sessions. The feet were positioned on the bench to avoid lumbar arching. During each repetition, the participants were required to perform the eccentric phase in a controlled manner and maintain a static position for $\sim 1 \mathrm{~s}$ at the end of this phase (i.e. bar resting on the chest) before performing the concentric phase at maximal intended velocity upon hearing the command. This pause was interposed between the eccentric and concentric phases to minimize the contribution 
of the rebound effect and allow for more reproducible measurements [32]. Throwing the bar at the end of the concentric phase was not allowed. Warm-up consisted of 5 min of upper-body joint mobilisation exercises, followed by a set of 6 repetitions with loads of 25 and $10 \mathrm{~kg}$ for men and women, respectively. The exact same warm-up and progression of submaximal loads up to the 1-RM was repeated in the post-test for each participant. After the warm-up, the initial load was set at $35 \mathrm{~kg}$ for men and $20 \mathrm{~kg}$ for women and progressively increased, by $10 \mathrm{~kg}$, until the attained MPV was $<0.4 \mathrm{~m} \cdot \mathrm{s}^{-1}$. Thereafter, each load was individually adjusted with smaller increments so that 1-RM could be precisely determined. The average number of attempts below $0.4 \mathrm{~m} \cdot \mathrm{s}^{-1}$ was $3.2 \pm 1.2$. Mean test velocity was defined as the mean of the MPV values, calculated at $5 \%$ increments from 30-100\%1-RM, and derived from the load-velocity data from each participant's progressive loading test.

For both exercises, the heaviest load that each participant could lift with correct technique while completing a full range of movement and without any external help was considered the participant's actual 1-RM. Three repetitions were executed for light ( $\leq 50 \% 1-\mathrm{RM})$, two for medium (50-80\% 1-RM) and only one repetition for the heaviest loads (>80\% 1$\mathrm{RM})$. Inter-set rests were $3 \mathrm{~min}$. The highest value for each velocity variable at each load was considered for subsequent analysis. A dynamic measurement system (T-Force System, Ergotech, Murcia, Spain), which consists of a linear velocity transducer interfaced to a computer by a 14-bit analog-to-digital data acquisition board and custom software, provided auditory and visual velocity feedback in real-time. Velocity was sampled at $1000 \mathrm{~Hz}$ and smoothed using a 4th order low-pass Butterworth filter with no phase shift and a $10 \mathrm{~Hz}$ cut-off frequency. Repeatability of the device and analysis methods have been reported elsewhere [33]. Strong verbal encouragement was provided during all tests to motivate participants to give maximal effort.

\section{Statistical analyses}

A second-order polynomial equation for predicting the corresponding MPV to each \%1RM was developed for men. The regression equation was cross-validated in women in the following manner; the regression equation derived from men (validation sample, $\mathrm{n}=$ 25) was applied to women (cross-validation sample, $n=25$ ) to obtain a predicted MPV for each participant. Thereafter, the women's sample was then divided into two groups, validation $(\mathrm{n}=13)$ and cross-validation $(\mathrm{n}=12)$ groups. Here, another equation was 
created from the validation group's data and was applied to the cross-validation group. Validity analyses included the calculation of a set of statistics aimed at providing information about the level of agreement and the magnitude of errors (both in absolute and relative values) incurred when comparing predicted and measured MPV [26]. The magnitude of errors was assessed by the standard error of measurement (SEM), which was calculated from the root mean square of the intra-individual total mean square to determine the amount of variability caused by prediction error [26]. Results are presented both in absolute $\left(\mathrm{m} \cdot \mathrm{s}^{-1}\right)$ and relative terms as a coefficient of variation $(\mathrm{CV}=100$ $\left.\mathrm{SEM} \cdot \mathrm{mean}^{-1}\right)$. The following statistics were used as indicators of agreement: The intraclass correlation coefficient (ICC), one-way random-effects $(1, \mathrm{k})$ model, and its $95 \%$ confidence interval (CI) were calculated according to Koo and Li's guidelines [34]. Lin's concordance correlation coefficient (CCC) was calculated to detect the agreement and systematic error between predicted and measured MPV by assessing how close their paired velocity outcomes were to the best-fit line, and how far this line was from the $45^{\circ}$ concordance line through the origin [27]. A CCC value of 1 represents perfect agreement, i.e., all the points lay exactly on the concordance line. Both the mean square deviation (MSD) and the variance of the difference between measurements (VMD) were used as error indicators. The closer the MSD is to zero the closer the predicted value to the measured value, since this indicates a constant and proportional systematic error and random error. Similarly, the closer the VMD is to zero the greater the precision (less dispersion of random error). Percent deviation from zero was also calculated for MSD and VMD. Linear regression analysis and Pearson's correlation coefficient (r) were used to assess the extent of the linear relationship between measured and predicted MPV values. Linear equations $(\mathrm{Y}=\mathrm{aX}+\mathrm{b})$ were fitted assuming that ideal values for the slope (a) should be close to 1 whilst the constant (b) should be close to zero to minimally alter the explanatory variable $(\mathrm{X})$. The standard error of the estimate (SEE) was calculated as the standard deviation of the residuals as a measure of variation around the regression line. The level of agreement between measured and predicted velocity from the different equations was also assessed using Bland-Altman plots and the calculation of systematic bias and its $95 \%$ limits of agreement (LoA = Bias \pm 1.96 standard deviation "SD") [35]. T1 and T2 absolute repeatability was assessed by the SEM which was expressed in relative terms through CV. Relative repeatability was assessed by the ICC with $95 \% \mathrm{CI}$ calculated with a one-way random effects model. According to Stokes [36], CV values of $\leq 15 \%$ can be classified as "satisfactory", whereas the ICC values were classified 
according to Fleiss [37] as an "excellent" (ICC $\geq 0.75)$, a "moderate to good" $(0.40<$ ICC $<0.75)$ or a "worse" (ICC $\leq 0.40)$ correlation. Differences between the sexes in strength and test characteristics were analysed using a one-way analysis of variance (ANOVA) and homogeneity of variance across sexes was verified using Levene's test. The differences in the load-velocity relationship between sexes were assessed using a 2 (sex) $\times 15$ (load) repeated measures ANOVA. When significant differences were observed a Bonferroni's post hoc comparison was performed. Significance was accepted at the $\mathrm{P} \leq$ 0.05 level. Statistical analyses were performed using a custom Microsoft Excel spreadsheet and SPSS software version 23.0 (SPSS, Chicago, IL, USA). Figures were designed using SigmaPlot 12.0 (Systat Software Inc, San Jose, California, USA).

\section{RESULTS}

For the SQ, $1-R M$ strength was $110.7 \pm 22.4 \mathrm{~kg}$ and $67.2 \pm 14.9 \mathrm{~kg}$ (i.e. $1.38 \pm 0.26$ and $1.14 \pm 0.26$ normalized per $\mathrm{kg}$ of body mass) in men and women, respectively. 1-RM strength for the BP was $81.0 \pm 13.0 \mathrm{~kg}$ and $40.8 \pm 9.1 \mathrm{~kg}$ (i.e. $1.00 \pm 0.17$ and $0.69 \pm 0.16$ normalized per kg of body mass) in men and women, respectively. All maximum strength values were statistically different between the sexes $(\mathrm{P}<0.05)$. Men performed more loads $(\mathrm{P}<0.05)$ during the incremental test up to the $1-\mathrm{RM}$ in both exercises $(\mathrm{SQ}: 7.9 \pm 1.6$; BP: $7.0 \pm 1.5$ ) than women (SQ: $6.7 \pm 1.1$, BP: $6.2 \pm 1.1$ ).

Second-order polynomial equations to predict the MPV attained with each \%1-RM in both SQ and BP were developed for men. These equations were cross-validated in women. Thereafter, second-order equations for predicting MPV values corresponding to each \%1-RM were developed for women (Figure 1).

\section{********* INSERT FIG $1 * * * * * * * * * * * *$}

Table 1 shows the results between MPV values (predicted and measured) for men (validation: predicted MPV from men's equation, $\mathrm{n}=25$ ), women (cross-validation: predicted MPV from men's equation, $n=25$ ), women (validation: predicted MPV from women`s equation, $n=13$ ) and women (cross-validation: MPV predicted from women's equation, $\mathrm{n}=12$ ) in the SQ and BP exercises. When the equation was applied in men (validation), there was a lower magnitude error and higher level of agreement than when the same equation was applied in women (cross-validation). However, when the women's equation (obtained from the women validation group) was used in the cross-validation 
women, the group magnitude of error and level of agreement were similar for both groups. Moreover, these values were similar to those observed for men when using their sexspecific equation.

\section{********* INSERT TABLE 1*************}

Figure 2 shows the scatter plots of predicted and measured velocity from men's equations applied to men (validation) and women (cross-validation), in the SQ and BP exercises. The best-fit regression lines together with the Bland-Altman plots for each comparison are shown. When the men's equation was applied to men (validation), it showed higher agreement and more regular variation in both SQ and BP than when the men's equation was applied to women (cross-validation). The men's equation applied in women (crossvalidation) showed a systematic bias in MPV of $\sim 0.08 \mathrm{~m} \cdot \mathrm{s}^{-1}$ and $0.02 \mathrm{~m} \cdot \mathrm{s}^{-1}$, for SQ and BP, respectively. Figure 3 shows the scatter plots of predicted and measured velocity from the women's equations applied to the women in the validation group and the women cross-validation group, for the SQ and BP exercises. When the women's equation was applied in both validation women group and cross-validation women group the bias was similar in both groups and exercises, and this bias was smaller and more distributed than when the men's equation was applied to women.

$* * * * * * *$ INSERT FIG $2 * * * * * * * * * * *$

******** INSERT FIG $3 * * * * * * * * * * *$ 


\section{Differences between the sexes in the load-velocity relationship}

The MPV values for each \%1-RM, for both men and women, were obtained from the individual's adjustments from approximately 30\% 1-RM onwards, in increments of 5\% for both SQ and BP (Table 2). A significant "sex $\times$ load" interaction was observed for SQ $(\mathrm{P}=0.04)$, whereas no significant "sex $\times$ load" interaction was observed for BP $(\mathrm{P}=0.26)$. There was a "sex" effect for both exercises ( $\mathrm{SQ}: \mathrm{P}<0.001, \mathrm{BP}: \mathrm{P}=0.02)$. ANOVA tests applied to the mean velocity attained at each tested \%1-RM revealed that men attained statistically higher values than women (P-range: $<0.001-0.05$ ). These differences between sexes were observed in light- to moderate-loads ( $\leq 75 \% 1-\mathrm{RM})$ in BP exercise, whereas in SQ exercise differences between sexes were observed up to $95 \% 1$ RM.

\section{********** INSERT TABLE $2 * * * * * * * * * * * *$}

Repeatability of the individualized load-velocity relationship in bench press and squat exercises

No significant differences were observed in 1-RM strength between T1 and T2 either in SQ (Men: $110.7 \pm 22.4$ and $111.1 \pm 22.2 \mathrm{~kg}$; Women: $67.2 \pm 14.9$ and $68.1 \pm 15.0 \mathrm{~kg}$ ) or in BP (Men: $81.3 \pm 12.8$ and $81.6 \pm 14.1 \mathrm{~kg}$; Women: $40.9 \pm 9.0$ and $41.8 \pm 9.5 \mathrm{~kg}$ ).

Reliability analyses were performed with the mean velocity test obtained from the individual's adjustments from approximately 30\% 1-RM onwards. Both men and women showed "excellent" repeatability values for SQ (Men: ICC=.908 (.790; .960), CV=3.8\%; Women: $\mathrm{ICC}=.931(.845 ; .969), \mathrm{CV}=3.7 \%)$. and BP (Men: $\mathrm{ICC}=.950(.887 ; .978)$, $\mathrm{CV}=4.5 \%$; Women: $\mathrm{ICC}=.852(.644 ; .946), \mathrm{CV}=4.8 \%)$.

Predicting relative load from velocity data

Since it is of practical importance to estimate the relative load (\%1-RM) from velocity measurements, then applying velocity as the independent variable, a prediction equation to estimate relative load (Load, \%1-RM) from velocity can be obtained:

General equations for SQ: 
Men: $\operatorname{Load}(\% 1 \mathrm{RM})=-27.966 \mathrm{MPV}^{2}-39.090 \mathrm{MPV}+113.119\left(\mathrm{R}^{2}=0.925 ; \mathrm{SEE}=\right.$ $5.99 \%)$

Women: Load $(\% 1 \mathrm{RM})=-42.196 \mathrm{MPV}^{2}-31.018 \mathrm{MPV}+112.937\left(\mathrm{R}^{2}=0.913 ; \mathrm{SEE}\right.$ $=5.68 \%)$

General equations for BP:

Men: $\operatorname{Load}(\% 1 \mathrm{RM})=-12.941 \mathrm{MPV}^{2}-51.895 \mathrm{MPV}+115.747\left(\mathrm{R}^{2}=0.927 ; \mathrm{SEE}=\right.$ $5.93 \%)$

Women: $\operatorname{Load}(\% 1 \mathrm{RM})=-84.293 \mathrm{MPV}^{2}-7.008 \mathrm{MPV}+113.309\left(\mathrm{R}^{2}=0.954 ; \mathrm{SEE}=\right.$ $4.06 \%)$

\section{DISCUSSION}

The present study examined the validity of using lifting velocity to estimate relative load during resistance exercise for both men and women, as well as analysed whether the strong association between \%1-RM and lifting velocity typically observed for men can be extrapolated to women. Additionally, this study examined the repeatability of these relationships in different exercises (BP and SQ) for each sex separately. The findings of this study confirm the assumption that the concentric bar velocity in BP and SQ exercises is a valid tool to estimate the relative load (\%1-RM) in both men and women. Although a better prediction can be obtained if a sex- (men and women) specific equation is used. As previously shown $[8,9,17,18]$, data suggest that lifting velocity allows us to determine, in real time, the actual relative intensity (\%1-RM) being performed. However, different equations should be used for each sex since men attained higher velocities against each \%1-RM, particularly with light and moderate loads, compared to women during BP and SQ exercises. Whether these differences are due to modifiable factors through training (e.g. strength level, sporting, and movement skill) rather than inherent sex differences should be further investigated [38].

Previous studies have attempted to validate the use of load-velocity relationship to estimate relative load during resistance exercise [7-9, 12-18]. Notwithstanding, most of these studies performed methods based on correlation, but these methods cannot, on its own, assess systematic bias [26]. In this study, we chose to use a comprehensive set of statistics aimed at assessing load-velocity relationship in two of the most traditional 
exercises in both men and women. The different statistical methods used as indicators of agreement showed high level of agreement for all equations (validation and crossvalidation). However, a lower magnitude of error and higher level of agreement was observed when a women-specific equation was applied in women Hence, although a general equation may be used to predict relative load in both men and women, a more accurate prediction could be obtained if a sex-specific equation is used. Moreover, the specific equation developed for women worked well for both validation and crossvalidation groups. As a result, our prediction equation could be applied to other women (i.e. future studies or during VBT sessions).

Close relationships were observed between movement velocity and \%1-RM in both men $\left(S Q: R^{2}=0.933 ; B P: R^{2}=0.920\right)$ and women (SQ: $\left.R^{2}=0.915 ; B P: R^{2}=0.954\right)$. However, men attained higher velocity values than women (P-range: $<0.001-0.05)$. Specifically in $\mathrm{SQ}$, men attained higher velocity values in almost all loads ( $\leq 95 \% 1-\mathrm{RM})$ than women, whereas in BP men lifted faster than women with light and moderate loads ( $\leq 75 \% 1-R M)$. These results are in line with the findings of previous studies conducted on horizontal and inclined BP, and seated military press exercises [19-21]. However, velocity against the heaviest loads did not significantly differ between the sexes, attaining the 1-RM at very similar velocity (SQ: $0.33 \pm 0.06$ vs. $0.30 \pm 0.06 \mathrm{~m} \cdot \mathrm{s}^{-1}$; BP: $0.16 \pm 0.05$ vs. $0.17 \pm 0.04$ $\mathrm{m} \cdot \mathrm{s}^{-1}$, in men and women respectively, Table 2). A practical implementation of these findings is that women seem to have higher strength deficit than men. This strength deficit should be understood as the percentage of maximal strength potential which is not used during a given motor task [39]. Consequently, the findings of the present study suggest the use of different equations for men and women in upper- and lower-body exercises such as BP and SQ. This comparison between men and women for the load-velocity relationship in SQ exercise, to the best of our knowledge, has not been previously reported in the literature. Therefore, although strong relationships between lifting velocity and \%1$\mathrm{RM}$ in the SQ exercise have already been reported $[15,17,18]$, this is the first study to show that this variable can also be used to precisely predict the actual \%1-RM in women.

Previous studies have shown high repeatability of the load-velocity relationship in BP [8] and bench-pull [40]. However, it should be noted that this is the first study comparing the repeatability of the load-velocity relationship in women. Remarkably, individual loadvelocity relationships showed to be consistent and stable for both sexes (ICC $>0.85$ and 
$\mathrm{CV}<5.0 \%$ ). Therefore, the findings of the present study support the use of load-velocity relationships for reliable and accurate determination of the actual \%1-RM being used in SQ and BP exercises, in both men and women, without the need to perform demanding, time-consuming and possibly fatiguing 1-RM or repetition to failure assessments. Strength and conditioning professionals now have a valuable tool for assessing strength, prescribing and monitoring the training load for their women athletes quickly and easily in BP and SQ, two of the most widely used exercises. Previous equations published in men are not necessarily valid for women, thus, different exercises that have been previously determined in men should be treated with caution or tested specifically in women before application (e.g. hip-thrust, leg-press). Given these observed sex differences, it is unclear how women would have responded to the same VBT program utilized for men in previous studies.

In conclusion, our findings confirm that movement velocity can be used to accurately prescribe the relative load (\%1-RM) regardless of sex in both upper-body and lower-body exercises. This study provides additional support for the necessity of developing more accurate prescription of the loads during VBT programs, instead of using general (men's) equations for women, since our results confirm that men and women attain different velocities against each \%1-RM, particularly with light and moderate loads, in BP and SQ. Therefore, when estimating load from velocity measures (or vice versa) it will be necessary to use the specific equation provided for each sex. Nevertheless, the velocity of the 1-RM did not differ significantly between sexes. Finally, the load-velocity relationship showed high repeatability. Future studies that wish to employ BP or SQ in their interventions in women now have reference data upon which to base their selection of VBT variables, which is an important methodological issue. Additionally, strength and conditioning professionals now have a valuable tool for assessing strength, prescribing and monitoring the training load for their women athletes quickly and easily in BP and SQ, two of the most widely used exercises.

\section{ACKNOWLEDGEMENTS}

The authors declare no conflict of interest.

\section{REFERENCES}

1. Suchomel TJ, Nimphius S, Bellon CR et al. The Importance of Muscular Strength: Training Considerations. Sports Med 2018; 48: 765-785. doi:10.1007/s40279-018-0862-z 
10.1007/s40279-018-0862-z [pii]

2. Kraemer WJ, Ratamess NA. Fundamentals of resistance training: progression and exercise prescription. Med Sci Sports Exerc 2004; 36: 674-688. doi:00005768200404000-00017 [pii]

3. Fry AC. The role of resistance exercise intensity on muscle fibre adaptations. Sports Med 2004; 34: 663-679. doi:34104 [pii]

4. Bird SP, Tarpenning KM, Marino FE. Designing resistance training programmes to enhance muscular fitness: a review of the acute programme variables. Sports Med 2005; 35: 841-851

5. Buckner SL, Jessee MB, Mattocks KT et al. Determining Strength: A Case for Multiple Methods of Measurement. Sports Med 2017; 47: 193-195. doi:10.1007/s40279-0160580-3

6. Scott BR, Duthie GM, Thornton HR et al. Training Monitoring for Resistance Exercise: Theory and Applications. Sports Med 2016; 46: 687-698. doi:10.1007/s40279-015-04540

7. Gonzalez-Badillo JJ, Marques MC, Sanchez-Medina L. The importance of movement velocity as a measure to control resistance training intensity. J Hum Kinet 2011; 29A: 1519. doi:10.2478/v10078-011-0053-6

jhk-29a-15 [pii]

8. Gonzalez-Badillo JJ, Sanchez-Medina L. Movement velocity as a measure of loading intensity in resistance training. Int J Sports Med 2010; 31: 347-352. doi:10.1055/s-00301248333

9. Loturco I, Pereira LA, Cal Abad CC et al. Using the Bar-Velocity to Predict the Maximum Dynamic Strength in the Half-Squat Exercise. Int J Sports Physiol Perform 2015. doi:2015-0316 [pii]

10.1123/ijspp.2015-0316. doi:2015-0316 [pii]

10.1123/ijspp.2015-0316

10. Chapman PP, Whitehead JR, Binkert RH. The 225-1b Reps-to-Fatigue Test as a Submaximal Estimate of 1-RM Bench Press Performance in College Football Players. The Journal of Strength \& Conditioning Research 1998; 12: 258-261

11. Niewiadomski W, Laskowska D, Gąsiorowska A et al. Determination and Prediction of One Repetition Maximum (1RM): Safety Considerations. 2008; 19: 109. doi:https://doi.org/10.2478/v10078-008-0008-8

12. Sanchez-Medina L, Gonzalez-Badillo JJ, Perez CE et al. Velocity- and power-load relationships of the bench pull vs. bench press exercises. Int J Sports Med 2014; 35: 209216. doi:10.1055/s-0033-1351252

13. Sanchez-Moreno M, Rodriguez-Rosell D, Pareja-Blanco F et al. Movement Velocity as Indicator of Relative Intensity and Level of Effort Attained During the Set in Pull-Up Exercise. Int J Sports Physiol Perform 2017; 12: 1378-1384. doi:10.1123/ijspp.2016-0791

14. Munoz-Lopez M, Marchante D, Cano-Ruiz MA et al. Load-, Force-, and Power-Velocity Relationships in the Prone Pull-Up Exercise. Int J Sports Physiol Perform 2017; 12: 12491255. doi:10.1123/ijspp.2016-0657

15. Conceicao F, Fernandes J, Lewis $\mathrm{M}$ et al. Movement velocity as a measure of exercise intensity in three lower limb exercises. J Sports Sci 2015. doi:10.1080/02640414.2015.1090010: 1-8. doi:10.1080/02640414.2015.1090010

16. de Hoyo M, Nunez FJ, Sanudo B et al. Predicting Loading Intensity Measuring Velocity in Barbell Hip Thrust Exercise. J Strength Cond Res 2019. doi:10.1519/JSC.0000000000003159. doi:10.1519/JSC.0000000000003159 
17. Sánchez-Medina L, Pallarés JG, Pérez CE et al. Estimation of Relative Load From Bar Velocity in the Full Back Squat Exercise. Sports Med Int Open 2017; 01: E80-E88. doi:10.1055/s-0043-102933

18. Martinez-Cava A, Moran-Navarro R, Sanchez-Medina L et al. Velocity- and power-load relationships in the half, parallel and full back squat. J Sports Sci 2019; 37: 1088-1096. doi:10.1080/02640414.2018.1544187

19. Balsalobre-Fernández C, García-Ramos A, Jiménez-Reyes P. Load-velocity profiling in the military press exercise: Effects of gender and training. International Journal of Sports Science \& Coaching 2018; 13: 743-750. doi:10.1177/1747954117738243

20. Torrejón A, Balsalobre-Fernández C, Haff GG et al. The load-velocity profile differs more between men and women than between individuals with different strength levels. Sports Biomechanics 2019; 18: 245-255. doi:10.1080/14763141.2018.1433872

21. García-Ramos A, Suzovic D, Pérez-Castilla A. The load-velocity profiles of three upperbody pushing exercises in men and women. Sports Biomechanics 2019. doi:10.1080/14763141.2019.1597155: 1-13. doi:10.1080/14763141.2019.1597155

22. Pestana-Melero FL, Haff GG, Rojas FJ et al. Reliability of the Load-Velocity Relationship Obtained Through Linear and Polynomial Regression Models to Predict the 1-Repetition Maximum Load. J Appl Biomech 2018; 34: 184-190. doi:10.1123/jab.2017-0266

23. Banyard HG, Nosaka K, Haff GG. Reliability and Validity of the Load-Velocity Relationship to Predict the 1RM Back Squat. J Strength Cond Res 2017; 31: 1897-1904. doi:10.1519/JSC.0000000000001657

24. Miller AE, MacDougall JD, Tarnopolsky MA et al. Gender differences in strength and muscle fiber characteristics. Eur J Appl Physiol Occup Physiol 1993; 66: 254-262. doi:10.1007/bf00235103

25. Sandbakk O, Solli GS, Holmberg HC. Sex Differences in World-Record Performance: The Influence of Sport Discipline and Competition Duration. Int J Sports Physiol Perform 2018; 13: 2-8. doi:10.1123/ijspp.2017-0196

26. Atkinson $\mathrm{G}$, Nevill AM. Statistical methods for assessing measurement error (reliability) in variables relevant to sports medicine. Sports Med 1998; 26: 217-238

27. Lin L, Hedayat AS, Sinha B et al. Statistical Methods in Assessing Agreement. Journal of the American Statistical Association 2002; 97: 257-270. doi:10.1198/016214502753479392

28. Harriss DJ, MacSween A, Atkinson G. Ethical Standards in Sport and Exercise Science Research: 2020 Update. Int J Sports Med 2019; 40: 813-817. doi:10.1055/a-1015-3123

29. Sanchez-Medina L, Perez CE, Gonzalez-Badillo JJ. Importance of the propulsive phase in strength assessment. Int J Sports Med 2010; 31: 123-129. doi:10.1055/s-0029-1242815

30. Jidovtseff $\mathrm{B}$, Croisier J, Scimar $\mathrm{N}$ et al. The ability of isoinertial assessment to monitor specific training effects. J Sports Med Phys Fitness 2008; 48: 55-64

31. Haff GG, Triplett NT. Essentials of Strength Training and Conditioning. 4th Edition Human kinetics 2015.

32. Pallares JG, Sanchez-Medina L, Perez CE et al. Imposing a pause between the eccentric and concentric phases increases the reliability of isoinertial strength assessments. J Sports Sci 2014; 32: 1165-1175. doi:10.1080/02640414.2014.889844

33. Sanchez-Medina L, Gonzalez-Badillo JJ. Velocity loss as an indicator of neuromuscular fatigue during resistance training. Med Sci Sports Exerc 2011; 43: 1725-1734. doi:10.1249/MSS.0b013e318213f880

34. Koo TK, Li MY. A Guideline of Selecting and Reporting Intraclass Correlation Coefficients for Reliability Research. J Chiropr Med 2016; 15: 155-163. doi:10.1016/j.jcm.2016.02.012

35. Bland JM, Altman DG. Statistical methods for assessing agreement between two methods of clinical measurement. Lancet 1986; 1: 307-310 
36. Stokes M. Reliability and Repeatability of Methods for Measuring Muscle in Physiotherapy. Physiotherapy Practice 1985; 1: 71-76. doi:10.3109/09593988509163853

37. Fleiss JL. The Design and Analysis of Clinical Experiment. John Wiley Sons, New York 1987; ISBN:9781118032923. doi:10.2307/2531561

38. Nimphius S. Exercise and Sport Science Failing by Design in Understanding Female Athletes. Int J Sports Physiol Perform 2019. doi:10.1123/ijspp.2019-0703: 1-2. doi:10.1123/ijspp.2019-0703

39. Siff M. Supertraining 5th ed Supertraining Institute 2000.

40. Garcia-Ramos A, Ulloa-Diaz D, Barboza-Gonzalez P et al. Assessment of the load-velocity profile in the free-weight prone bench pull exercise through different velocity variables and regression models. PLoS One 2019; 14: e0212085. doi:10.1371/journal.pone.0212085

PONE-D-18-31875 [pii]

\section{FIGURE LEGENDS}

Fig. 1 Relationships between relative load (\%1RM) and bar velocity for the full back squat (upper panel) and bench press (lower panel) exercises. Data obtained from raw loadvelocity values derived from the progressive loading tests performed on the sample of 25 men and 13 women. Solid lines show the fitted curve to the data. Black circles represent men and grey circles represent women. MPV: mean propulsive velocity. SEE: standard error of estimate.

Fig. 2 Level of agreement in mean propulsive velocity (MPV) between measured and predicted velocity obtained from men equation being applied to men (validation) and women (cross-validation) in the squat (upper panel) and bench press (lower panel) exercises. Linear regression (left panel) and Bland-Altman plots (medium and right panels) are shown. Black circles represent men and grey circles represent women. SEE: standard error of estimate. SD: standard deviation. LoA: Level of agreement.

Fig. 3 Level of agreement in mean propulsive velocity (MPV) between measured and predicted velocity obtained from women equation being applied to women validation group and women cross-validation group in the squat (upper panel) and bench press (lower panel) exercises. Linear regression (left panel) and Bland-Altman plots (medium and right panels) are shown. Black circles represent men and grey circles represent women. SEE: standard error of estimate. SD: standard deviation. LoA: Level of agreement. 


\section{TABLE LEGENDS}

Table 1 Agreement between measured and predicted velocity values in the squat and bench press exercises.

Table 2 Estimated mean propulsive velocity values for each \%1-RM in the squat and bench press exercises for men $(n=25)$ and women $(n=25)$ derived from the individual loadvelocity relationships. 